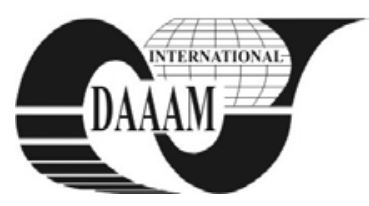

\title{
DIGITIZING SYSTEM ATOS - MEASURING TURBO COMPRESSOR HOUSING
}

\author{
BOGOVIC, I[va] - N[icol]; BARISIC, B[ranimir]; KATALINIC, B[ranko]; \\ KRSULJA, M[arko] \& CAR, Z[latan]
}

\begin{abstract}
This paper shows the measurement and control of digitization technology. Measuring of turbo compressor housing is shown with digitalizing ATOS system, measurement procedure is explained, all features and additional functions of this measurement system were described. Software package used in investigation is explained. Recommendations are made for using this technology, measuring procedure and measurement results of turbo commpresed housing have been given.
\end{abstract}

Key words: ATOS, 3D scanner, measuring, digitization, camera

\section{INTRODUCTION}

In recent years optical-digitizing measuring technologies have been used more and more in the science and industry.

The development of optical 3D shape measurement methods is rapidly gaining importance as it represents the main competition and alternative to CMM - Coordinate measuring systems. It can give fast measurement, there are no limitations of working area and it can be used in field inspection.

New optical digitizing measuring systems could be used for the high-end 3D digitizing, optical 3D deformation analysis, forming analysis, the optical coordinate measuring machines, the precise positioning, motion and deformation calculation, measurement of surface and shapes inspection.

The focus of this paper will be on Advanced TOpometric Optical Sensors as optical based 3D scanning systems designed to acquire accurate scan data of three-dimensional objects which goal is digitizing measurement and shapes inspection.

It delivers three-dimensional measurement data for industrial components such as sheet metal parts, tools and dies, turbine blades, prototypes, injection molded and casted parts. Instead of measuring single points, full part geometry is captured in a dense point cloud or polygon mesh describing the object's surface and primitives precisely.

3D digitizing with this scanner delivers for different object sizes and complexity (www.gom.com, 2011):

- Highly accurate 3D coordinates

- $\quad$ Full-field deviation to CAD (Computer-aided design)

- Section-based analysis

- Complete measuring reports.

\section{ATOS - HIGH RESOLUTION, OPTICAL 3D SCANNER}

\subsection{Triple Scan - Revolutionary scanning technique}

Using this brand new technology the Triple Scan produces a high accuracy and improved measurement of shiny surface, complete data on complex components with deep pockets or fine edges such as turbine blades, reducing the number of individual scans and resulting in a simple handling.

\subsection{Blue Light Technology - scan independently of environmental light}

Triple Scan is also equipped with blue light technology. The narrowband blue light enables precise measurements to be carried out independently of environmental lighting conditions (Fig. 1. ATOS - Advanced TOpometric Sensor).

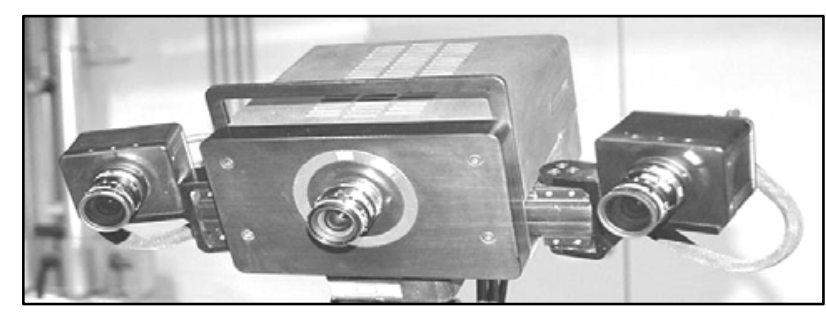

Fig. 1. ATOS (www.cimos.eu, 2011)

\subsection{High Resolution 3D-Scanner}

The accuracy, measurement resolution and measuring area are completely adaptable to the application requirements. This allows for the highest resolution for highly detailed, small parts with measuring volumes down to $38 \mathrm{~mm}$, or for extremely fast digitizing of large objects with measuring volumes up to $2 \mathrm{~m}$.

\subsection{Measuring of FT- IR spectra in transmission mode}

Measuring technique in transmission mode focuses on detection of IR ray passing through the sample placed in a liquid cuvette (see Fig. 2). The sample is applied to the cuvette with a syringe.

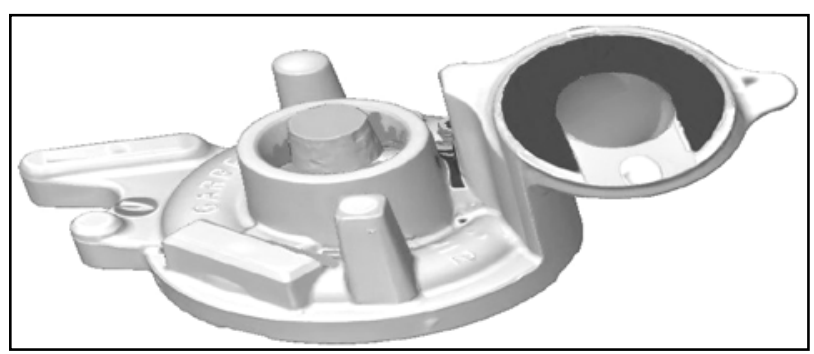

Fig. 2. The results of digitalization the measured turbo compressor housings

\subsection{Industrial advantages}

$3 \mathrm{D}$ scanner is an accurate and cost-effective solution in a number of different application areas including:

- Quality Control

- $\quad$ Reverse Engineering

- $\quad$ Rapid Prototyping

- $\quad$ Rapid Milling

- Digital Mock-Up.

3D scanners have been produced and constantly developed since 1995 and with more than 2500 installations in measurement and analysis rooms as well as factory and production halls worldwide, it has been for a long time an accepted measurement method beside the mechanical measurement machines (www.3d-skeneri.com, 2011).

The ATOS sensor combines high data quality in short measurement time with flexibility and stability for industrial environments. 


\subsection{Touch Probe}

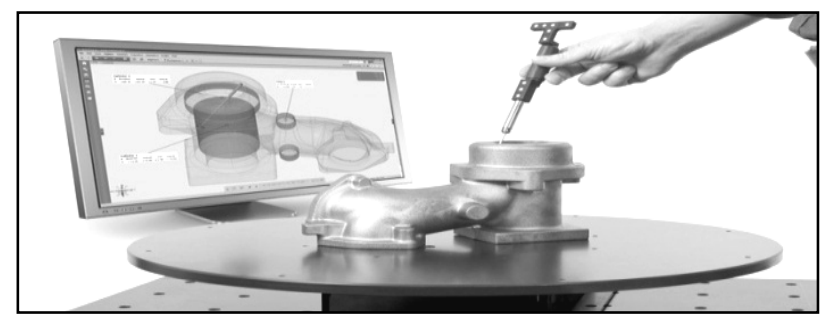

Fig. 3. Touch Probe

The Touch Probe combines full-field and touch probe 3D measurement. It allows quick measurement in difficult to access areas, comparison directly to CAD, measurement of primitives, quick measurement of individual points and online alignment.

\section{SOFTWARE}

The ATOS Professional software is used to run the sensor head, to process the 3D point cloud and to edit and post-process the data. The simple graphical user interface helps support today's demanding tasks in quality control, manufacturing processes and reverse engineering. ATOS Professional features include: sensor control, polygon mesh generation and editing, sectioning, feature and character line detection, primitive generation are basic software functions. For quality control and result analysis, tools such as CAD data import; import of measuring plans; registration; full-field deviation plots; sectionbased analysis; deviation of individual points; calipers, angles and diameters; report creation are available (www.topomatika.hr, 2011).

\section{PRINCIP OF MEASURING}

In order to measure the entire geometry of complex objects, reference targets are attached to the object and their coordinates are determined by photogrammetry. These targets define the object coordinate system in the particular referent relative sensor to object position. Typical types of sensors consisted of projector and the two cameras provide over-determined mathematical triangulation model. Fundamental problem with structured light projecting technology is in the correspondence problem, since to obtain triangulation points one needs to locate for each pixel in left image $\mathrm{m}$ the corresponding pixel in the right image. Projector purpose is there just to provide the unique definition of matchable object points, hence for a dual camera system projector doesn't necessarily has to be calibrated (www.bib.irb.hr, 2011).

\section{MEASUREMENT PROCESS}

The head of ATOS is mounted on a stand and positioned in front of the measuring turbo compressor housing and in that way positioning of sensor have been made. After that follows the scanning i.e. projecting different fringe patterns onto the object's surface by means of a white light projection unit and capturing these patterns by 2 CCD cameras at either side of the sensor head. From these images the software based on the optical transformation equations calculates the 3D coordinates of over 4 million determined points.

The current sensor position has been automatically determined by the system and transforms the single measurements into a general coordinate system. The calibration using the photogrammetric methods of the system has been derived during each measurement, thus ensuring detailed and speed up measuring under the hard industrial conditions. The reference points for the system are targets pointed to the object.
By means of the reference points the transformation into the general coordinate system has been done.

Presented subject has been scanned segment by segment. Measuring surfaces were covered with titan-oxide powder and referent points were positioned on turbo compressor housing. Single measurements are automatically matched in one integrality i.e. common coordinate system by means of referent points (white circles on black background).

After scanning, the calculating of a complete highresolution polygon mesh of the object was done, small triangles in curved and large triangles in flatter areas without diminishing the mesh's accuracy were made. At the end of digitizing a polygon mesh of the object surface has been obtained. Further result processing like mesh editing or reconstruction of surfaces depend on specific tasks and should always be discussed with respect to the actual application.

\section{CONCLUSION}

In this paper, a description of the optical digitalizing system ATOS is made, and the measurement of turbocharger housing. Digitizing gave us computer model of the followed object which was used for the analysis of functional coordinates of the product.

During measuring the tolerances and the various factors that influence the occurrence of errors were observed. Things that cause errors are weather conditions, the method of setting measuring tape, the selection of reference points, the number of selected views and shots (used 2 to look at 40 frames).

The rules of measurement system are explained and ATOS measurement procedure used in the company Cimmos Buzet were presented. The resulting digitized polygonized images was compared with the final 3D model. The deviation of the obtained values and the CAD model is shown, based on experiences upper tolerance should be $3 \mathrm{~mm}$. On the measured specimen was not found significant deviations from the required tolerance. Additional already introduced CAD models in the future can be compared with the original model with the aim of checking the measurements obtained in production.

This analysis gave an answer to the required precision machining of individual parts of the jig manufacturing turbocharger housings.

For future research, it is necessary that optical methods for digitizing shapes begin to research the topic of how the system functioned with 3 or more cameras that would have the possibility of achieving variable external calibration parameters.

\section{ACKNOWLEDGEMENTS}

The authors would like to acknowledge the support provided by the National CEEPUS Office of Croatia and National CEEPUS Office Austria, which helped the research through mobility in the frame of the CEEPUS II HR 0108 project.

\section{REFERENCES}

*** (2011) www.cimos.eu - Private documents of Cimos company Buzet, Croatia, Accessed on: 2011-04-05

*** (2011) www.gom.com - Optical Measuring Techniques, Accessed on: 2011-04-13

*** (2011) www.topomatika.hr - 3D scanning, 0ptical measuring systems and computer processing, Accessed on: 2011-06-20

*** (2011) www.3d-skeneri.com - Obitelj ATOS 3D skenera, Accessed on: 2011-06-25

*** (2011) www.bib.irb.hr - A new 3d scanning-aided technology in tool design (D. Semenski, A. Bakić, A. Marinov, N. Drvar), Accessed on: 2011-06-14 